\title{
Concentration of angiopoietins 1 and 2 and their receptor Tie-2 in peripheral blood in patients with chronic obstructive pulmonary disease
}

\author{
Dorota Kierszniewska-Stępień ${ }^{1}$, Tadeusz Pietras ${ }^{1}$, Maciej Ciebiada², Paweł Górski ${ }^{1}$, Henryk Stępień ${ }^{3}$
}

\author{
'Department of Pneumonology and Allergy, Medical University of Lodz, Lodz, Poland \\ Head of the Department: Prof. Paweł Górski MD, PhD \\ 2Department of General and Oncological Pneumonology, Medical University of Lodz, Lodz, Poland \\ Head of the Department: Prof. Adam Antczak MD, PhD \\ ${ }^{3}$ Department of Immunoendocrinology, Medical University of Lodz, Lodz, Poland \\ Head of the Department: Prof. Henryk Stępień MD, PhD
}

Postep Derm Alergol 2015; XXXII (6): 443-448

DOI: $10.5114 /$ pdia.2014.44008

\begin{abstract}
Introduction: Both angiopoietins (angiopoietin 1 - Ang-1, angiopoietin 2 - Ang-2) and angiopoietin receptors (Tie) are involved in angiogenesis and vascular remodeling.

Aim: To assess concentrations of Ang-1, Ang-2 and Tie- 2 in blood of patients with chronic obstructive pulmonary disease (COPD) and evaluate if their concentrations depend on the severity of the disease.

Material and methods: Thirty patients with COPD (stage II-IV) and 8 healthy smokers as well as 8 healthy nonsmokers were included in the study. Detailed history was taken, physical examination and spirometry tests were done and blood samples were taken for evaluation of serum concentrations of Ang-1, Ang-2 and Tie.

Results: Among COPD patients, 8 patients suffered from moderate disease, 8 patients had severe, while 14 patients had very severe disease. The concentrations of Ang-1 and Ang-2 were not significantly greater in patients with COPD than in healthy controls. The highest concentrations of Ang-1 and Ang-2 were observed in patients with moderate COPD, and levels of Ang-2 correlated with Tie-2 in this group of patients. The levels of Ang- 1 were the lowest in healthy non-smokers and in patients with severe COPD, where they inversely correlated with Tie-2. The concentrations of Ang-2 were not significantly higher in patients with moderate COPD when compared with those with severe and very severe disease and healthy smokers, and were significantly higher than in healthy non-smokers.

Conclusions: It is possible that Ang-1, Ang-2 and Tie-2 play an important role especially in the early stage of COPD but not in the late phase when vascular complications of the disease occur.
\end{abstract}

Key words: disease severity, vascular bed, complications, angiogenesis, receptor.

\section{Introduction}

Chronic obstructive pulmonary disease (COPD) is a common respiratory disease which affects about 10\% of the general population. Primarily COPD was thought to affect exclusively the respiratory system. However, it is commonly known that there is a systemic aspect of chronic inflammation in COPD, which contributes to the development of extrapulmonary changes, like vascular complications, which are often observed in these patients. Vascular complications seen in COPD patients result from the phenomenon of angiogenesis and remodeling of the microvascular bed. Angiogenesis means the formation of new blood vessels on the basis of the existing ones, whereas microvascular remodeling is often the structural change of the vessel (thickening of the arterioles, capillaries and venules) without creating a new one. Both these phenomena can occur simultaneously or independently of each other if they are activated by various stimuli [1, 2]. These processes lead to pulmonary hypertension, a characteristic COPD feature. According to current knowledge, the von Euler mechanism consti-

Address for correspondence: Maciej Ciebiada MD, PhD, Department of General and Oncological Pneumonology, Medical University of Lodz, 22 Kopcinskiego St, 90-153 Lodz, Poland, phone: +48 42 678 21 29, 48426776699 , fax: +48 42 678 2129 , e-mail: maciej_ciebiada@op.pl Received: 17.12.2013, accepted: 31.01.2014. 
tutes the basis for this phenomenon. The results of recent studies suggest that one of the factors co-responsible for the development of pulmonary hypertension and possibly also for the remodeling of the vascular bed could be vascular endothelial growth factor (VEGF) discovered by Dvorak [3, 4].

Hypoxia is the main stimulus of neovascularization. Not only does it stimulate angiogenesis, but also accounts for remodeling of blood vessels in such diseases as asthma and COPD [5]. In response to hypoxia, various cytokines stimulate the formation of new blood vessels. Angiogenesis is an important well-coordinated process regulated by many angiogenic factors. The main cytokine initiating neoangiogenesis is VEGF, the expression of which occurs under the influence of the hypoxia-inducible factor (HIF-inducible factor hypoxia). Angiogenesis is initiated by increasing vascular permeability and vascular extension. The VEGF, initially called the vascular permeability factor, stimulates also the proteolytic enzymes and regulates expression of the receptor cells involved in vascular remodeling while protecting endothelial cells [4-6]. The next step concerns the maturation of newly occurred vessels. Cross-linking of endothelial cells among themselves and also with the extracellular matrix and mesenchymal matrix makes this stage important. This process involves platelet derived growth factor (PDGF) which, as a mitogen, is responsible for the recruitment of mesenchyme [7, 8].

Angiopoietins (Ang-1, Ang-2) and receptors with properties of tyrosine kinase (Tie-1, Tie-2) are involved in the late phase of angiogenesis. Their essential function is to connect endothelial cells with mesenchymal cells. Tie-1 receptor participates in the endothelial cell differentiation. By contrast, Tie-2 receptor has a dual role. On one hand, it is crucial for creating a network of blood vessels, i.e. angiogenesis, and on the other hand it maintains the integrity of these vessels $[9,10]$. Angiopoietins (Ang-1, Ang-2), which have different ligands for Tie-2 through Tie-2, can stimulate or inhibit vascular endothelial cells. Angiopoietin 1 (Ang-1), as a proangiogenic protein, contributes to the formation, maturation and stabilization of the vascular network. By contrast, angiopoietin 2 (Ang-2) - involved in vascular remodeling, sensitizes endothelial cells to the angiogenic factors. The blockage of the Tie-2 receptor by Ang-1 contributes to the destabilization of the newly created vessels by the loss of adventitia and smooth muscle cells [11, 12].

\section{Aim}

Since both angiopoietins (Ang-1, Ang-2) and angiopoietin receptors (Tie-1, Tie-2) may be responsible for angiogenesis and remodeling of the microvascular bed in chronic obstructive pulmonary disease, we wanted to assess their concentrations in blood of patients with COPD and healthy smokers and non-smokers and to evaluate if the concentrations of Ang-1, Ang-2 and Tie depend on the severity of the disease.

\section{Material and methods}

\section{Patients}

The study comprises a group of 30 patients with COPD: 12 women and 18 men aged 50-87 (mean: 65.7 \pm 1.9 ). The diagnosis of COPD was based on the Global Initiative for Chronic Obstructive Pulmonary Disease (GOLD 2010) criteria [13]. The patients recruited in the outpatient clinic had been clinically stable for at least 4 weeks before the first visit. None of them had either exacerbation or changed the dose of medications in the last 3 months. We excluded the following patients: patients who had had an airway infection for 4 weeks prior to the preliminary visit and in the course of the study, those receiving systemic glucocorticosteroids for 4 weeks prior to the preliminary visit and in the course of the study, as well as patients with a history of malignancy, tuberculosis, autoimmune disease and asthma.

According to GOLD standards [13], all the patients were divided into 3 groups: those with moderate $(n=8)$, severe $(n=8)$ and very severe COPD $(n=14)$.

The healthy subjects were recruited from the general population. The control groups comprised 8 healthy smokers with an average 25 pack-year (20-30 pack-year) and 8 healthy non-smokers.

After the informed consent was obtained, all the participants underwent physical examination, spirometry. Blood samples were collected from each subject.

The project was approved by the Ethical Committee for Scientific Studies of the Medical University of Lodz, RNN/4/12/KE.

\section{Pulmonary function tests}

Spirometry was performed in each participant in the morning by the same technician before breakfast using the Lungtest 1000 spirometer (MES, Krakow, Poland). The patients had to withdraw bronchodilators before the test. Pulmonary function values were expressed as a percentage of predicted values. Measured parameters included: forced expiratory volume in the $1^{\text {st }} \mathrm{S}\left(\mathrm{FEV}_{1}\right)$ (\% predicted) and $\mathrm{FEV}_{1} / \mathrm{FVC}$ (forced expiratory volume in the $1^{\text {st }} \mathrm{s}$ to forced vital capacity).

\section{Blood sampling and analyses}

Blood samples were taken from the antecubital vein in the morning between 7:00 and 8:00 AM after an overnight fast. The blood was processed within $1 \mathrm{~h}$ after the collection and serum was aliquotted and stored at $-70^{\circ} \mathrm{C}$ until the analysis. The measurements of serum concentration of Ang-1, Ang-2 and Tie-2 were conducted using commercial, enzyme-linked immunoassay kits (R\&D (ELISA) Systems Inc., 614 McKinley Place NE, Minneapolis 
Table 1. The patients' baseline characteristics

\begin{tabular}{lccccc}
\hline Parameter & \multicolumn{3}{c}{ COPD patients } & \multicolumn{2}{c}{ Healthy controls } \\
\cline { 2 - 6 } & Stage II & Stage III & Stage IV & Smokers & Non-smokers \\
\hline$N$ & 8 & 8 & 14 & 8 & 8 \\
\hline Gender, female : male & $4: 4$ & $3: 5$ & $5: 9$ & $4: 4$ & $4: 4$ \\
\hline Age & $67.0 \pm 5.37$ & $70.8 \pm 4.43$ & $63.9 \pm 3.6$ & $53.7 \pm 5.2$ & $54.2 \pm 2.6$ \\
\hline FEV $(\%)$ & $60.6 \pm 3.2$ & $44.1 \pm 8.9$ & $34.5 \pm 14.1$ & $100 \pm 10.1$ & $103 \pm 9.1$ \\
\hline
\end{tabular}

Values presented as mean $\pm S D$. $N$-number of patients.

MN 55413, USA). The serum detectable range of Ang-1 was $14.272-65.570 \mathrm{pg} / \mathrm{ml}$ (mean: $37.122 \mathrm{pg} / \mathrm{ml}$ ) and the sensitivity of assay was less than $1.36 \mathrm{pg} / \mathrm{ml}$. The intraassay coefficient of variability (CV) is $2.4 \%$ and inter-assay CV $-6.4 \%$. The serum detectable range of Ang-2 was 1065-8707 pg/ml (mean: 2494 pg/ml) and the sensitivity of assay was less than $1.20 \mathrm{pg} / \mathrm{ml}$. The intra-assay CV was $6.9 \%$ and inter-assay CV $-10.4 \%$. The serum detectable range of Tie-2 is 18.6-75.3 ng/ml (mean: $27.4 \mathrm{ng}$ / $\mathrm{ml}$ ) and the sensitivity of assay was less than $0.001 \mathrm{ng} /$ $\mathrm{ml}$. The intra-assay CV was $4.6 \%$ and inter-assay CV was $8.3 \%$

\section{Statistical analysis}

All values are presented as mean \pm SEM. Data distribution was checked on the basis of the Shapiro-Wilk test and to compare the groups with normally distributed data, the Student's t-test was used, whereas for not normally distributed data, the Mann Whitney U-test was performed. Value of $p<0.05$ was considered as significant.

\section{Results}

The baseline characteristics of the COPD patients and healthy controls are shown in Table 1. In total, there were 30 COPD patients and 16 healthy patients. Of those with COPD, 8 (26.7\%) patients suffered from moderate disease, 8 (26.7\%) patients had severe COPD while 14 (46.6\%) patients had very severe disease. Healthy patients were divided into two groups according to their smoking status: smokers $(n=8)$ and non-smokers $(n=8)$ (Table 1). The patients with very severe COPD had significantly smaller FEV values, longer history of disease and smoked cigarettes longer than patients with severe and moderate disease, whereas in patients with moderate COPD, FEV 1 was smaller and the history of the disease as well as history of smoking was longer than in patients with moderate COPD (Table 1).

\section{Concentration of angiopoietin 1}

The concentration of Ang-1 was greater in patients with COPD than in healthy controls, however, the difference was not significant. The greatest concentration of Ang-1 was observed in patients with moderate COPD

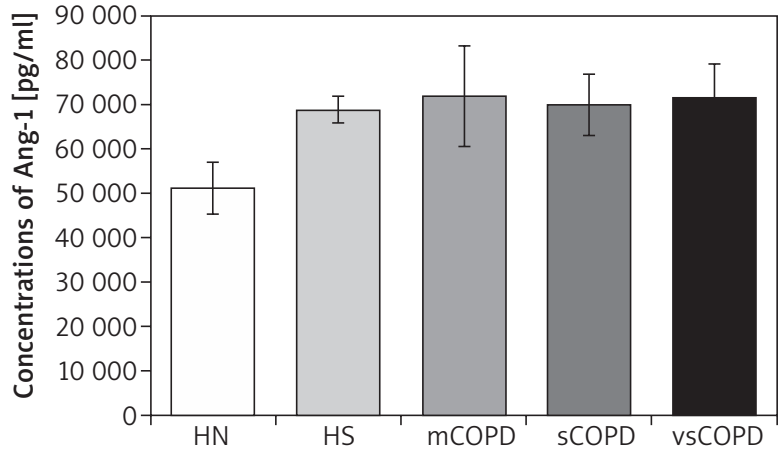

Figure 1. Concentrations of Ang-1 in peripheral blood of patients with moderate, severe and very severe COPD and in healthy controls. Values presented as mean \pm SEM

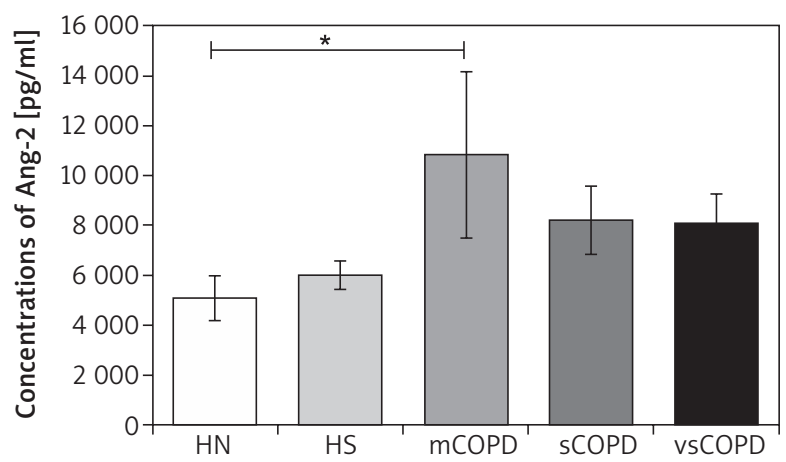

Figure 2. Concentrations of Ang-2 in peripheral blood of patients with moderate, severe and very severe COPD and in healthy controls. Values presented as mean \pm SEM

$(71987.5 \pm 27937.5 \mathrm{pg} / \mathrm{ml})$, whereas the lowest in healthy non-smokers $(p>0.05)$ (Figure 1$)$.

\section{Concentration of angiopoietin 2}

The concentration of Ang-2 was higher in patients with COPD than in healthy controls. The highest concentration of Ang-2 was observed in patients with moderate COPD (10843.3 $\pm 3333.3 \mathrm{pg} / \mathrm{ml})$, although it was not significantly higher when compared with concentrations observed in patients with severe and very severe COPD and healthy smokers, and was significantly higher than in healthy non-smokers (5093.3 \pm 896.7 pg/ml) (Figure 2). 


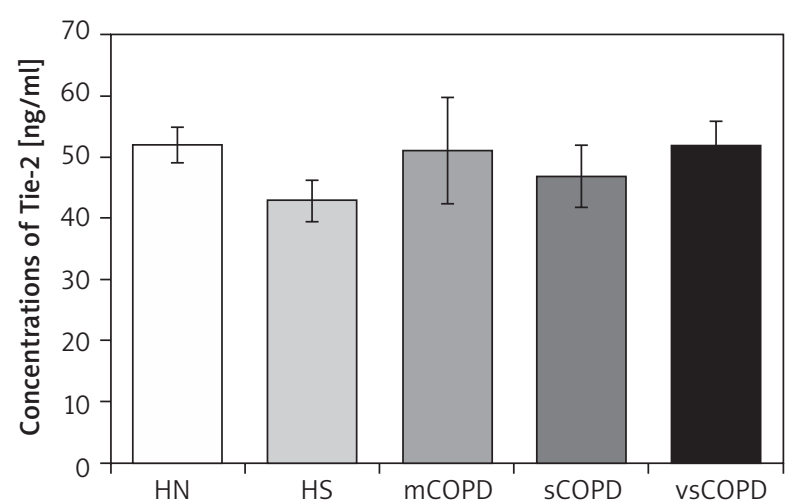

Figure 3. Concentrations of Tie-2 in peripheral blood of patients with moderate, severe and very severe COPD and in healthy controls. Values presented as mean \pm SEM

\section{Concentration of Tie-2}

The highest concentration of Tie-2 receptor was detected in the control group of non-smokers $(52.0 \pm 2.9 \mathrm{ng} / \mathrm{ml})$, however, no significant differences were found between groups of patients and between the control group of smokers and non-smokers (Figure 3).

\section{Correlations}

In healthy smokers, serum concentrations of Ang2 correlated inversely with concentrations of Ang-1 $(r=-0.51, p=0.18)$. Furthermore, in patients with moderate COPD, concentrations of Tie-2 correlated positively with concentrations of Ang-2 ( $r=0.64, p=0.14)$. In patients with severe COPD there was a negative correlation between serum Tie-2 and Ang- 1 levels ( $r=-0.68$, $p=0.05)$ and negative correlation between the levels of Tie-2 and Ang-2 ( $r=-0.77, p=0.018)$. In addition, a positive correlation between serum Ang-2 and the patients' age $(r=0.71, p=0.04)$, and between serum levels of Ang-2 and Ang-1 $(r=0.62, p=0.08)$ were observed in this group of patients. There was a positive correlation between serum concentrations of Tie- 2 and Ang-2 $(r=0.49$, $p=0.036)$ and the concentrations of Ang-2 and Ang-1 $(r=0.41, p=0.08)$ in patients with very severe COPD.

\section{Discussion}

Our study showed the highest concentrations of Ang-2 in patients with moderate COPD. Contrary to our expectations, the concentrations of Ang-2 in groups of patients with severe and very severe COPD were similar to the controls. The observed phenomenon shows that the biochemical processes of COPD begin in the initial stage, which initiates vascular remodeling, as reflected by the activity of angiopoietins. In advanced COPD, characterized by pulmonary hypertension and right heart failure, vascular remodeling is completed, hence the low concentration of Ang-2. This is only a hypothesis, how- ever, our earlier publication, which assessed the levels of VEGF and its soluble receptors (sVEGFR1, sVEGFR2) in the serum of patients with COPD, showed a significant increase in VEGF levels in patients with mild COPD [14]. Thus, these results indirectly confirmed our hypothesis. Surprisingly, there were no differences in angiopoietin concentrations and the receptor both in healthy smokers and non-smokers. Santos et al. [15], who immunohistochemically examined lung tissue samples taken during surgery, showed a significant increase in both VEGF expression and VEGF mRNA in patients with mild COPD and in healthy smokers who had not developed impaired ventilation yet. However, it is worth noticing that angiopoietins are the late mediators of angiogenesis and it is possible that the increase in VEGF concentration precedes the increase in concentrations of angiopoietins. The increase in the concentration of Ang-2 is also described in patients during exacerbation of COPD, as noted by Cho et al. [16, 17]. The concentration of Ang-2 correlated positively with CRP levels and negatively with $\mathrm{PaO} 2$. Similar observations were also confirmed by Oh et al. who showed that both hypoxia and VEGF up-regulate the concentration of Ang-2 in bovine microvascular endothelial cells $[16,17]$.

Other authors have shown that hypoxia induces expression of the gene for angiopoietins and for inflammatory cytokines $[18,19]$. It is worth remembering that in the course of COPD, the local hypoxia may occur, which may be caused by heterogeneous distribution of ventilation and perfusion. In patients with moderate COPD, there are some areas in the lungs which are hypoxic, despite normal levels of oxygen in the bloodstream. In the moderate stage of COPD we have also observed a positive correlation between serum Ang-2 and Tie-2, which may demonstrate a parallel gene expression of the mediator and its receptor. However, in more severe COPD, the concentration of Ang-2 and Tie-2 correlated negatively, which reflects the receptors' down-regulation. It is possible that Ang-2 in the advanced stage of COPD is not responsible for stimulation and progression of COPD. The obtained data suggest that Ang-2 constitutes a factor of vascular remodeling in COPD at the moderate stage.

There are important observations concerning the negative correlations between the concentrations of Ang- 1 and Tie-2 levels and between serum Ang-2 and concentration of Tie-2 in patients with severe COPD.

Surprisingly, there was no difference in the concentrations of Ang-1 between all the groups of patients with COPD. There have been no studies concerning Ang- 1 in the pathogenesis of vascular lesions in COPD. Nevertheless, our results suggest that Ang-1 does not play a significant role in the pathogenesis of COPD nor in the pathogenesis of vascular lesions in the course of COPD. This indirectly suggests a different genetic regulation of gene expression for Ang-1 and Ang-2. 
In the severe stage of COPD, there was a positive correlation between the serum Ang-2 and patients' age, which was not found in other groups. This phenomenon could be explained by the fact that in smoking patients subjected to high exposure to tobacco smoke, who had been indentified for higher concentrations of Ang-2, we could observe a well-developed collateral circulation in the cardiovascular system. This meant the prevention of cardiac death in this group of patients. However, it is exclusively our hypothesis, which is not supported by data from other studies.

There was also a positive correlation between serum Ang-2 and Tie-2 receptor in a very severe stage of COPD. This can be explained by the fact that in some patients, an increased expression of Ang-2 and Tie-2 receptor may be an additional factor stimulating angiogenesis and tumorigenesis. Studies have shown that COPD is an independent predictor of lung cancer. The average risk of developing lung cancer in this group of patients is 4 (from 2.7 to -5 ), with the average risk of 2.23 for men and 3.94 for women [20]. Jassem et al. have shown that the incidence of lung cancer in smokers is related to gene polymorphism, while Galvan and Dragani have confirmed a link between nicotine $15 q 25$ locus and the occurrence of lung cancer [21-23]. There is a certain group of patients with very severe COPD, in whom there is simultaneous activation of the expression of both Ang-2 and Tie-2. Such patients are probably at an increased risk of vascular remodeling and development of cancer, but this phenomenon requires further investigation and confirmation. The receptor for Ang-2 exhibits tyrosine kinase activity suggesting that it may act as a pro-oncogenic factor, as confirmed by Niedzwiecki in follicular thyroid carcinoma. The observed phenomenon may partially explain the coexistence of COPD and lung cancer. In all the groups of patients in our study, except for those with severe COPD, levels of Tie-2 receptor correlated with the concentration of angiopoietins (Ang-2), while in severe COPD such a correlation was negative. This phenomenon may be explained by receptors' down-regulation due to strong stimulation of the receptor by ligand, but it is unclear why it is observed exclusively in advanced COPD. Perhaps at this stage of this disease there are other mechanisms which account for the regulation of the receptor's expression.

What makes our study incomplete is the fact that we did not include the patients in the first stage of COPD (which was specified in GOLD guidelines). This was because somatic symptoms occurring in this group of patients are rarely reported and they do not seek specialized medical care but in Poland they are treated by family doctors.

\section{Acknowledgments}

The study was done in the Department of Pneumonology and Allergy and Department of Immunoendocrinology, Medical University of Lodz, Poland.
The study was self-funded (Medical University of Lodz) and was approved by the ethics committee of the Medical University in Lodz.

\section{Conflict of interest}

The authors declare no conflict of interest.

\section{References}

1. McDonald DM. Angiogenesis and remodeling of airway vasculature in chronic inflammation. Am J Respir Crit Care Med 2001; 164: 39-45.

2. Walsh DA, Pearson Cl. Angiogenesis in the pathogenesis of inflammatory joint and lung diseases. Arthritis Res 2001; 3: 147-53.

3. Ferrara N. Role of vascular endothelial growth factor in the regulation of angiogenesis. Kidney Int 1999; 56: 794-814.

4. Senger DR, Galli SJ, Dvorak AM, et al. Tumor cells secrete a vascular permeability factor that promotes accumulation of ascites fluid. Science 1983; 219: 983-5.

5. Forsythe JA, Jiang BH, lyer NV, et al. Activation of vascular endothelial growth factor gene transcription by hypoxiainducible factor 1. Mol Cell Biol 1996; 16: 4604-13.

6. Ferrara N, Keyt B. Vascular endothelial growth factor. Basic biology and clinical implication. EXS 1997; 79: 209-32.

7. Gupta K, Kshirsagar S, Li W, et al. VEGF prevents apoptosis of human microvascular endothelial cells via opposing effects on MAPK/ERK and SAPK/JNK signaling. Exp Cell Res 1999; 247: 495-504.

8. Sato N, Beitz JG, Kato J, et al. Platelet-derived growth factor indirectly stimulates angiogenesis in vitro. Am J Pathol 1993; 142: 1119-30.

9. Maisonpierre PC, Suri C, Jones PF, et al. Angiopoietin-2, a natural antagonist for Tie2 that disrupts in vivo angiogenesis. Science 1997; 277: 55-60.

10. Sato TN, Tozawa Y, Deutsch U, et al. Distinct roles of the receptor tyrosine kinases Tie-1 and Tie-2 in blood vessel formation. Nature 1995; 376: 70-4.

11. Wong AL, Haroon ZA, Werner S, et al. Tie2 expression and phosphorylation in angiogenic and quiescent adult tissues. Circ Res 1997; 81: 567-74.

12. Asahara T, Chen D, Takahashi T, et al. Tie2 receptor ligands, angiopoietin-1 and angiopoietin-2, modulate VEGF-induced postnatal neovascularization. Circ Res 1998; 83: 233-40.

13. Global Initiative for Chronic Obstructive Pulmonary Disease (GOLD) 2010. From the Global Strategy for the Diagnosis, Management and Prevention of COPD. Available from: http://www.goldcopd.org/.

14. Kierszniewska-Stepień D, Pietras T, Górski P, Stepien H. Serum vascular endothelial growth factor and its receptor level in patients with chronic obstructive pulmonary disease. Eur Cytokine Netw 2006; 17: 75-9.

15. Santos S, Peinado VI, Ramirez J, et al. Enhanced expression of vascular endothelial growth factor in pulmonary arteries of smokers and patients with moderate chronic obstructive pulmonary disease. Am J Respir Crit Care Med 2003; 167: 1250-6.

16. Cho YJ, Ma JE, Yun EY, et al. Serum angiopoietin-2 levels are elevated during acute exacerbations of COPD. Respirology 2011; 16: 284-90.

17. Oh H, Takagi H, Suzuma K, et al. Hypoxia and vascular endothelial growth factor selectively up-regulate angiopoietin-2 in bovine microvascular endothelial cells. J Biol Chem 1999; 274: 15732-9. 
18. Nizet TA, van den Elshout FJ, Heijdra YF, et al. Survival of chronic hypercapnic COPD patients is predicted by smoking habits, comorbidity, and hypoxemia. Chest 2005; 127 : 1904-10.

19. Parikh SM, Mammoto T, Schultz A, et al. Excess circulating angiopoietin-2 may contribute to pulmonary vascular leak in sepsis in humans. PLoS Med 2006; 3: e46.

20. Young RP, Hopkins RJ, Christmas T, et al. COPD prevalence is increased in lung cancer, independent of age, sex and smoking history. Eur Respir J 2009; 34: 380-6.

21. Jassem E, Szymanowska A, Siemińska A, Jassem J Palenie tytoniu a rak płuca. Pneumonol Alergol Pol 2009; 77: 469-73.

22. Galvan A, Dragani TA. Nicotine dependence may link the 15 q25locus to lung cancer risk. Carcinogenesis 2010; 31: 331-3.

23. Pietras T, Szemraj J, Witusik A, et al. The sequence polymorphism of MnSOD gene in subjects with respiratory insufficiency in COPD. Med Sci Monit 2010; 16: 427-32. 